\title{
Design and Analysis of Stacked Power Amplifier in Series-Input and Series-Output Configuration
}

\author{
Ming-Fong Lei, Student Member, IEEE, Zuo-Min Tsai, Member, IEEE, \\ Kun-You Lin, Member, IEEE, and Huei Wang, Fellow, IEEE
}

\begin{abstract}
The stacked-device power-combining technique is a proven method to increase the output power and load impedance of a power amplifier (PA) simultaneously. The series-input configuration is physically realizable for multicell stacked device configuration in monolithic circuits. The series-input and series-output stack configuration is rigorously analyzed and proven to increase both the input impedance and output impedance simultaneously, easing the matching circuit designs in high PAs. The effects of asymmetry of the input feed and amplifier cells due to distributed effects and process variation on the performance of the stack amplifier are discussed. A four-cell HBT amplifier operating at 5-6 GHz is demonstrated to validate the circuit concept.
\end{abstract}

Index Terms-Monolithic microwave integrated circuit (MMIC) power amplifiers (PAs), stacked amplifier.

\section{INTRODUCTION}

$\mathbf{P}$ OWER amplifiers (PAs) are the most demanding components in a transceiver system, as they occupy a very large chip/board area, require a lot of dc power, need heat dissipation, and demand good efficiency and linearity during operation. In order to deliver the most power, the device in a PA is matched to a load that allows the maximum voltage and current swing, rather than for optimum gain [1]. The idea of maximum power from maximum voltage and current swing is obvious, which can be easily explained with a dc- $I V$ curve and load-line theory. The load-pull simulation/measurements often used in RF designs is an extension of the load-line concept, and is elegantly explained in [2]. The power match point is usually low impedance relative to the system impedance, making it difficult to match.

To increase the output power of a PA, either the voltage swing or current swing of the amplifier needs to be increased. To increase the output power of the amplifier, one usually increases the size of the device periphery to allow a larger current swing. An increase in device size will increase its maximum power, but also decreases its optimum load impedance, which will be difficult to match. In a $50-\Omega$ system, a typical power device, which

Manuscript received May 5, 2007; revised August 19, 2007. This work was supported in part by the National Science Council of Taiwan, R.O.C., under Grant NSC 95-2219-E-002-009, Grant NSC 95-2219-E-002-006, Grant NSC 95-2752-E-002-003-PAE, and Grant NSC 95-2218-E-002-057 and by the National Taiwan University under Excellent Research Project 95R0062-AE00-01.

The authors are with the Graduate Institute of Communication Engineering and Department of Electrical Engineering, National Taiwan University, Taipei 106, Taiwan, R.O.C. (e-mail: kunyou@ntu.edu.tw; hueiwang@ew.ee.ntu.edu. tw).

Color versions of one or more of the figures in this paper are available online at http://ieeexplore.ieee.org.

Digital Object Identifier 10.1109/TMTT.2007.909147 is created by parallel combination of many smaller devices, will have an optimum load of less than $5 \Omega$, which is quite difficult to match; any further parallel combination for more power will render the matching circuit increasingly difficult to realize on-chip. Furthermore, the increase in dc current will require wider metals to satisfy current density rules. This means that for a high-power PA, the output matching components are often realized off-chip, and the chip layout itself will be quite bulky, all adding to the overall cost production. Hence, the parallel combination of devices can only increase the output power to a certain extent.

Rather than increasing output power by increasing current, one can combine the device in series and increase the voltage. The advantage of series connection is increased optimum load, making the device easier to match to system impedance. The cascode configuration shown in Fig. 1(a) is an excellent example of such implementation, and is ubiquitous in CMOS PA designs [3]-[5] and millimeter-wave frequencies [6], taking advantage of its output power and higher gain. Other series configurations are less common, but also can be seen in the literature. A three-device totem pole configuration, which is like a triple-cascode configuration, demonstrated a $36-\mathrm{dBm}$ output power at 4 $\mathrm{GHz}$ [7]. Another method connects all the devices' drains and sources in series, but the inputs and outputs are still connected to the devices in parallel, with proof-of-concept designs in $C$ - and $X$-band [8]. The HIFET/HiVP concept [9], of which the devices are connected in series, and the inputs are fed by shunt resistors; a wideband 2-W HIFET amplifier was demonstrated using MESFET [10], as well as a 20-dBm CMOS demonstration [11]. In another approach, the devices are stacked in series, but individually biased using dc blocks between the drain and sources of adjacent devices and RF chokes [12]. In the transformer-coupled stacking approach shown in Fig. 1(b), the devices' drain and source are stacked in series, and uses transformers to couple the input signals into the circuit; the stacked devices' current and voltage swing in unison to provide a larger output [13], [14]. A one- and two-cell monolithic study has been demonstrated [13] with results consistent with the theory.

A modification of the transformer-coupled stack has recently been implemented in a 2- $\mu \mathrm{m}$ HBT [15], shown in Fig. 1(c). Rather than feeding the input in parallel in the previous approach [13], [14], the input feed of the stack is also configured in series. Such a configuration has the additional advantages of increasing input impedance, as well as easier design and layout, since the methods of connection of input and output is identical, and one only needs to complete the design and layout of one cell, and connect them in succession. In this paper, the 

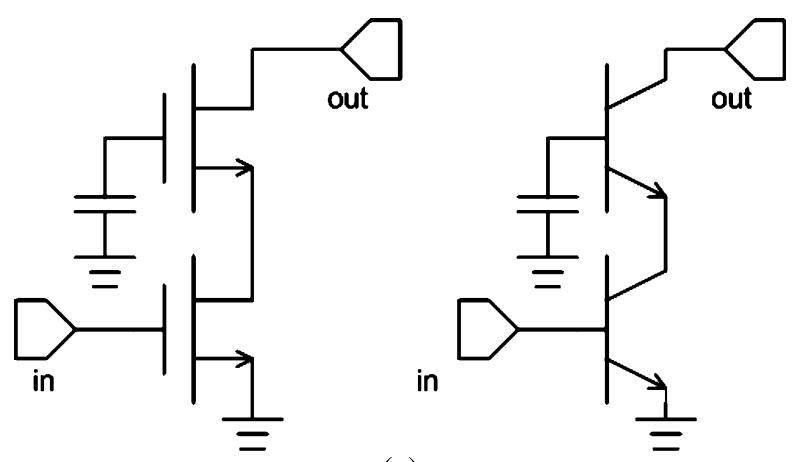

(a)

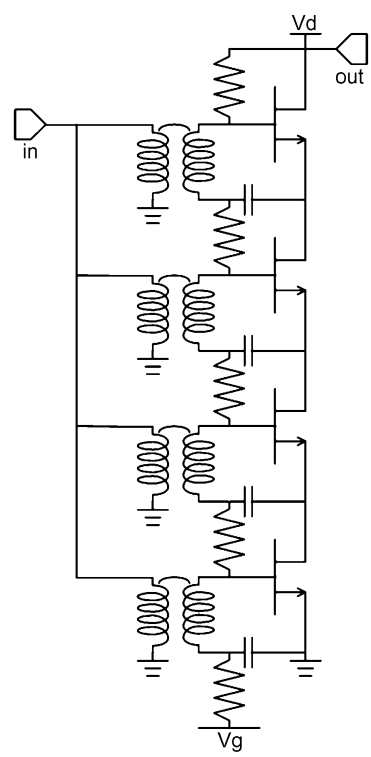

(b)

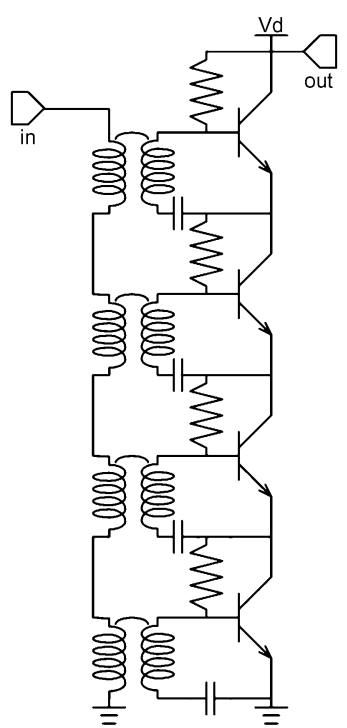

(c)
Fig. 1. Different types of stacked amplifiers. (a) Cascode configuration. (b) Transformer-coupled stack with parallel input feed. (c) Transformer coupled stack with series-input feed.

series-input and series-output transformer-coupled stacked PA will be investigated in detail. The working principles will be rigorously derived, and the nonlinear effects discussed. In particular, the distributed effect and component variations will affect the symmetry of the cell outputs, which will have an effect on the combination efficiency, gain, and the linearity performance of the stack amplifier. Such nonideal effects are observed in the four-cell HBT stack amplifier demonstration, and are in good correlation with simulation results. The performance of the four-cell HBT stack amplifier with actual modulation signals is evaluated.

\section{SERIES-InPut AND SERIES-OUtPut Stack}

In the original transformer-coupled stacked PA [13], the input is connected in parallel and the output is connected in series. The linear analysis proved that a stack of $n$ devices may be treated as a single device with output impedance increased by factor $n$, drain-to-source breakdown voltage increased by factor $n$, and input impedance decreased by factor $n$. The intermodulation distortion measurements show that the stack configuration does

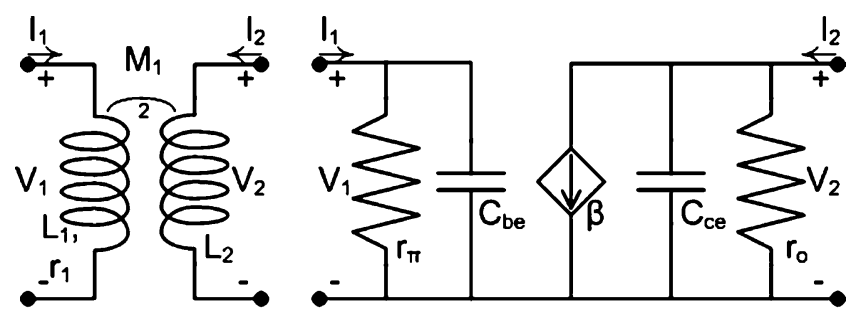

(a)

(b)

Fig. 2. (a) Equivalent model of a transformer. (b) Small-signal model of a unilateral bipolar transistor.

not generate further third-order intermodulation distortion signals, i.e., linearity is not compromised.

Here, the series-input and series-output stack is analyzed in detail. The linear analysis will show that this new configuration has an equivalent circuit to a single device with an increase in input impedance, output impedance, and breakdown voltage. The additional advantage of increased input impedance is very useful, as the input match in PA designs is just as difficult as the output match. Effects such as distributed effect and device variations, will affect the combination performance of the stack, the various effects may be summarized as magnitude and phase imbalance between adjacent cells, and its effects can be quantitatively discussed. The magnitude and phase imbalance of the cells will affect the linearity performance of the stack amplifier, and can be both simulated and measured.

This section contains three subsection. Section II-A presents the linear analysis of the series-input and series-output transformer coupled stacked amplifier. Section II-B discusses the reduction of gain due to the asymmetry of the cells. Section II-C presents the effects of physical elements on the linearity of the stacked amplifier.

\section{A. Linear Analysis}

The cell of the transformer-coupled stack is composed of two components: a coupling transformer and transistor. To simplify the analysis, the transformer is assumed to be lossy only at the input port, and a unilateral bipolar model is used. The bias network is then assumed to be open circuits, and dc blocks between the base and the emitter terminals are assumed to be short. The $Z$-parameters for a two-port lossless transformer are defined as

$$
\left[\begin{array}{l}
V_{1} \\
V_{2}
\end{array}\right]=\left[\begin{array}{cc}
j \omega L_{1}+r_{1} & j \omega M_{12} \\
j \omega M_{12} & j \omega L_{2}
\end{array}\right]\left[\begin{array}{l}
I_{1} \\
I_{2}
\end{array}\right]
$$

where $L_{1}$ is the self-inductance of the input port, $r_{1}$ is the input coil's resistance, $L_{2}$ is the self-inductance of the output port, $M_{12}$ is the mutual inductance, and the current points into the transformer, as shown in Fig. 2(a). The small-signal equivalent circuit of the bipolar model is shown in Fig. 2(b). An equivalent circuit of an $n$-element series-input series-output transformer-coupled stack is shown in Fig. 3. Since the transformer's characteristics are in the form of a $Z$-matrix, a test current signal is used for the analysis. A test current signal $I_{\text {in }}$ is applied into the input. It is assumed that all the transistors and transformers 


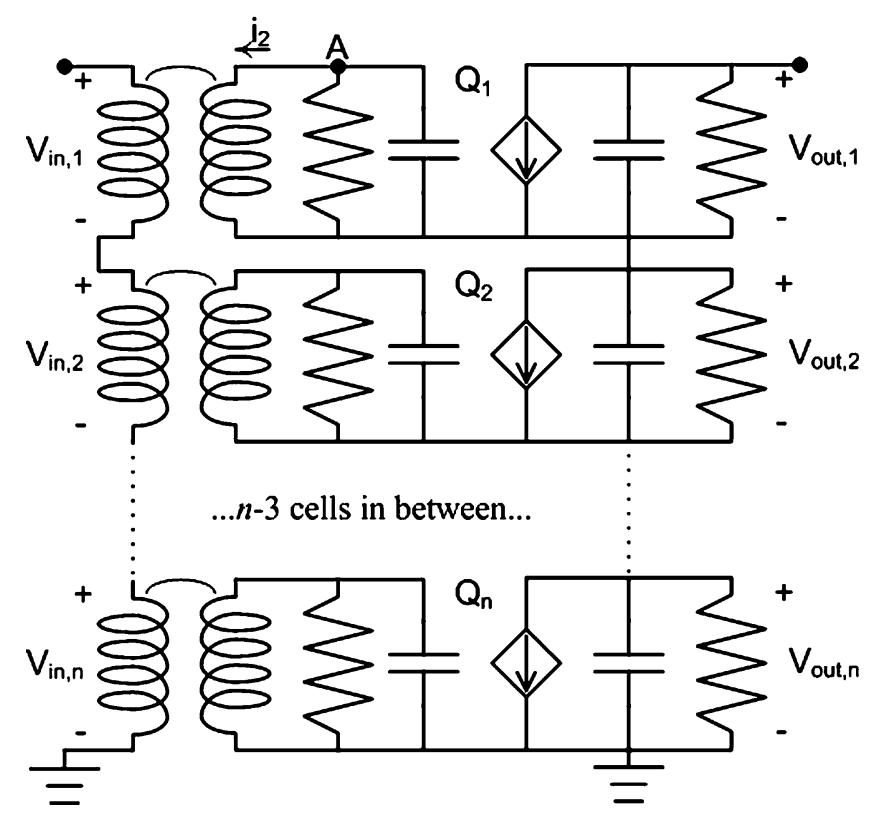

Fig. 3. Small-signal circuit of an $n$-cell series-input and series-output transformer coupled stack.

are identical. Starting from the top device $Q_{1}$, from (1), the voltages across the two coils of the transformers are

$$
\begin{aligned}
& V_{i n, 1}=\left(j \omega L_{1}+r_{1}\right) I_{\mathrm{in}}+j \omega M_{12} i_{2} \\
& V_{\mathrm{be}, 1}=j \omega M_{12} I_{\mathrm{in}}+j \omega L_{2} i_{2} .
\end{aligned}
$$

At node $\mathrm{A}$, the output coil of the transformer, and $C_{\mathrm{be}}$ and $r_{\pi}$ of the transistor should conform to Kirchoff's voltage law (KVL), therefore, the current $i_{2}$ can be expressed as

$$
-i_{2}=\left(j \omega C_{\mathrm{be}}+1 / r_{\pi}\right) \cdot V_{\mathrm{be}, 1} .
$$

Using (3) in (4), the current $i_{2}$ can be expressed in terms of the input current $I_{\text {in }}$. Reapplying $i_{2}$ to (2) and (3) will solve the voltages in terms of the input current $I_{\text {in }}$. Proceeding to the other cells, it is clear that in each cell, the output coils, $C_{\mathrm{be}}$ and $r_{\pi}$ form a closed loop, therefore, all of the input coils of the transformers have the same equation, and all of the output coils of the transformers have the same equation. The smallsignal collector currents of each cell will also be identical so the collector-emitter capacitance $C_{\mathrm{ce}}$ and early resistance $r_{o}$ can be considered as a series connection with a total impedance of $n \cdot\left[r_{o} / /\left(1 / j \omega \cdot C_{\mathrm{ce}}\right)\right]$ driven by a common emitter current. The total input voltage $V_{\text {in }}$ will be the sum of the voltages across each transformer, and the output voltage is

$$
\begin{aligned}
& V_{\text {out }}=-n \cdot g_{m} \cdot\left(j \omega M_{12}+\frac{\omega^{2} M_{12} L_{2}\left(j \omega C_{\mathrm{be}} r_{\pi}+1\right)}{r_{\pi}+j \omega L_{2}\left(j \omega C_{\mathrm{be}} r_{\pi}+1\right)}\right) \\
& \times I_{\text {in }}\left(\frac{r_{o}}{1+j \omega C_{\mathrm{ce}} r_{o}}\right) \text {. }
\end{aligned}
$$

From (6) and (8), the voltage gain is

$$
\begin{array}{r}
G_{V}=-g_{m} \frac{\left(j \omega M_{12}+\frac{\omega^{2} M_{12} L_{2}\left(j \omega C_{\mathrm{be}} r_{\pi}+1\right)}{r_{\pi}+j \omega L_{2}\left(j \omega C_{\mathrm{be}} r_{\pi}+1\right)}\right)}{\left(j \omega L_{1}+\frac{\omega^{2} M_{12}^{2}\left(j \omega C_{\mathrm{be}} r_{\pi}+1\right)}{r_{\pi}+j \omega L_{2}\left(j \omega C_{\mathrm{be}} r_{\pi}+1\right)}+r_{1}\right)} \\
\times\left(\frac{r_{o}}{1+j \omega C_{\mathrm{ce}} r_{o}}\right)
\end{array}
$$

The input impedance is

$$
Z_{\text {in }}=n \cdot\left(j \omega L_{1}+\frac{\omega^{2} M_{12}^{2}\left(j \omega C_{\mathrm{be}} r_{\pi}+1\right)}{r_{\pi}+j \omega L_{2}\left(j \omega C_{\mathrm{be}} r_{\pi}+1\right)}+r_{1}\right) .
$$

By applying a test voltage across the output and setting the input to short, the output impedance can be calculated. The transformers are assumed not stored with any energy, and the output impedance is simply

$$
Z_{\mathrm{out}}=n \cdot\left(\frac{r_{o}}{1+j \omega C_{\mathrm{ce}} r_{o}}\right)
$$

Defining the coupling factor of the transformer as

$$
K_{T}=\frac{\left(j \omega M_{12}+\frac{\omega^{2} M_{12} L_{2}\left(j \omega C_{\mathrm{be}} r_{\pi}+1\right)}{r_{\pi}+j \omega L_{2}\left(j \omega C_{\mathrm{be}} r_{\pi}+1\right)}\right)}{\left(j \omega L_{1}+\frac{\omega^{2} M_{12}^{2}\left(j \omega C_{\mathrm{be}} r_{\pi}+1\right)}{r_{\pi}+j \omega L_{2}\left(j \omega C_{\mathrm{be}} r_{\pi}+1\right)}+r_{1}\right)}
$$

the voltage gain of (9) can be conveniently expressed as

$$
G_{V}=-g_{m} K_{T}\left(\frac{r_{o}}{1+j \omega C_{\mathrm{ce}} r_{o}}\right) .
$$

From the analysis flow and the conclusions of (6)-(10), the series-input and series-output transformer coupled stack can be interpreted as a stack of common emitter devices with the same characteristics operating in unison. The small-signal voltage gain is the same as a single-device common emitter amplifier, albeit with a transformer coupling factor since the input signal is not directly connected to the transistor input. An $n$-cell series-input and series-output coupled stack also has the advantages of increased input impedance, and increased output impedance, compared to a single device. The increase in input impedance is an advantage since the input impedance of a power device is also quite low, and it is easier to match if it is also increased. The transformer coupling factor may also be used to tune the input match. The advantages of a series-input series-output stack also hold true with other configurations, i.e., if the cell is a cascode gain stage. The comparison between the series-input and series-output stack to various types of stack configurations is listed in Table I.

The above analysis can also be applied for FET devices with the same conclusions. If the input current is applied in the opposite direction, i.e., if the stack is fed from the bottom device first, the result is the same, except that the voltage gain has 
TABLE I

COMPARISON OF SHUNT COMBINATION, HiVP/HIFET, PARALLEL-INPUT STACK, AND SERIES-INPUT STACK TO SingLE DEVICE

\begin{tabular}{lcccc}
\hline \hline & $\begin{array}{c}\text { Input } \\
\text { Impedance }\end{array}$ & $\begin{array}{c}\text { Output } \\
\text { Impedance }\end{array}$ & $\begin{array}{c}\text { Voltage } \\
\text { (bias and } \\
\text { swing) }\end{array}$ & $\begin{array}{c}\text { Current } \\
\text { (bias and } \\
\text { swing) }\end{array}$ \\
\hline $\begin{array}{l}\text { Shunt } \\
\text { Combination }\end{array}$ & $1 / n$ & $1 / n$ & 1 & $n$ \\
$\begin{array}{l}\text { HiVP/HIFET } \\
{[9]}\end{array}$ & $--*$ & $n$ & $n$ & 1 \\
$\begin{array}{l}\text { Parallel-Input } \\
\begin{array}{l}\text { Stack [19] } \\
\text { Series-Input } \\
\text { Stack (this work) }\end{array}\end{array}$ & $1 / n$ & $n$ & $n$ & 1 \\
\hline \hline
\end{tabular}

* Not derived in this paper.

an opposite sign. The Miller effect may be used to take into account the base-collector capacitance. The input impedance can be adjusted by tuning $L_{1}$ for a simpler input match to the system impedance, while keeping other parameters rather constant. From the transformer efficiency (9), the finite loss of the transformer will reduce the gain of the amplifier, and should be minimized to maintain the stacked amplifier's gain.

\section{B. Effects of Phase and Magnitude Imbalances Between Cells on Combination Efficiency}

The linear analysis in Section II-A is based on the assumption that all the components are identical and are lumped in nature. In reality, process parameter gradients will cause variations between the devices and passive components across the wafer, and components are distributed in nature, resulting in phase and magnitude imbalances in each cell. These imperfections will impact the performance of the stack, specifically on the combination efficiency and the power performance. The effects are analyzed in detail.

The series-input series-output stack amplifier can be visualized as a series connection of $n$ amplifier blocks, as shown in Fig. 4. Each amplifier cell consists of a transformer and a transistor, as well as the biasing circuits and base-emitter dc blocking capacitances. The voltage gain of each amplifier cell is $A_{v}$. In the case where all amplifier cells are identical and driven in the linear range, the voltage gains should be the same. When all of the amplifier cells are identical, applying an input voltage of $V_{\text {in }}$ will result in an output voltage of $A_{v} \cdot V_{\text {in }}$, and the output power is $\left(A_{v} \cdot V_{\text {in }}\right)^{2} /\left(2 \cdot R_{L}\right)$.

Due to various reasons, the cells in a stack amplifier are not completely identical. Due to distributed effects of the transformers, the magnitude and phase at the input of the transistor in each cell is not always identical. There will be a slight phase delay and a magnitude drop as the signal progresses along the transformer ladder. The trace lines connecting the device collector to the emitter of the next device will also add a phase delay to the output voltage of each cell. The device in each cell may not be completely identical due to process variations, which will introduce phase and magnitude difference to the voltage gain between different cells. Such variations between the different cells can be summarized as an overall complex factor of

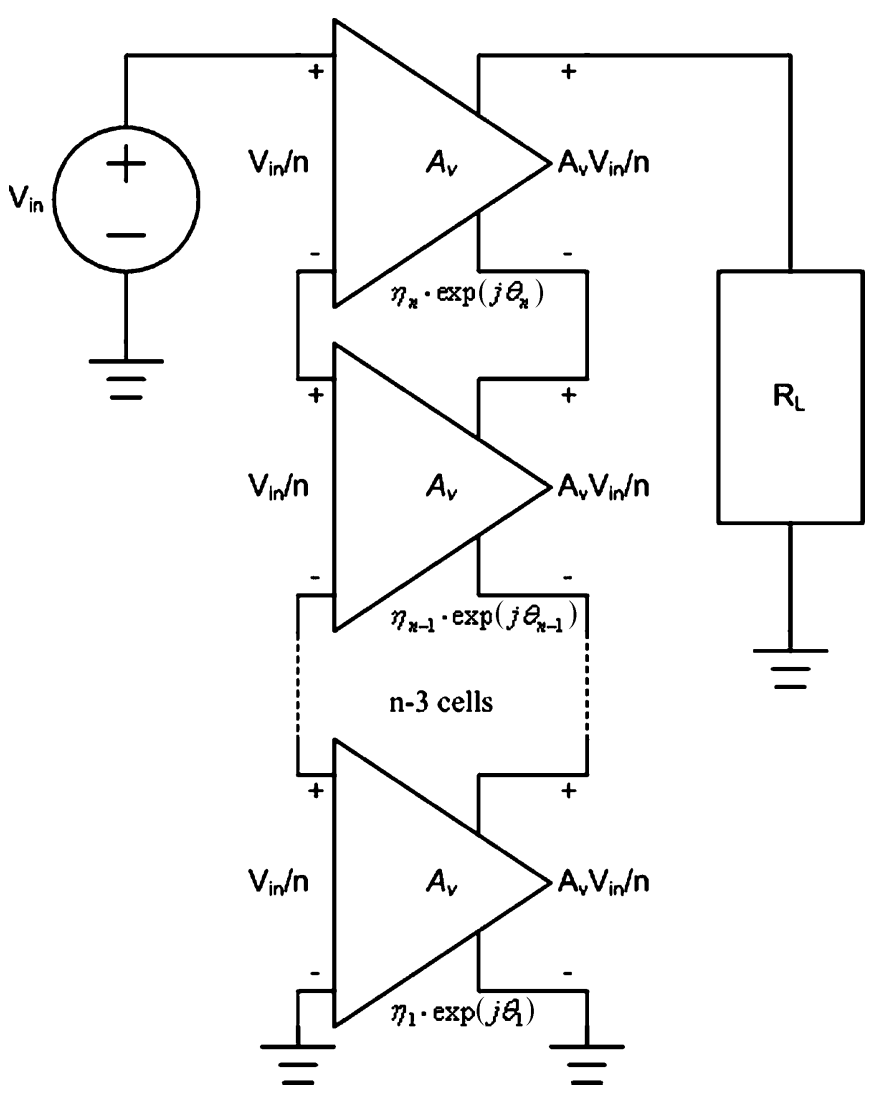

Fig. 4. Block diagram of an $n$-cell series-input series-output stack amplifier. Each amplifier block has the same voltage gain $A_{v}$ with different magnitude and phase variation expressed as $\eta_{i} \cdot \exp \left(j \theta_{i}\right)$.

$\eta_{i} \cdot \exp \cdot\left(j \cdot \theta_{i}\right), \eta_{i}$ is the magnitude factor, and $\exp \left(j \cdot \theta_{i}\right)$ is the phase; the subscript $i$ represents the cell number. Typically, $\eta$ will be less than unity and progressively smaller along the transformer ladder, representing an energy loss as the input signal propagates, but in some cases, the magnitude factor can be higher than unity, such as when a device has particularly higher gain than average. The phase factor $\theta$ will progressively increase along the transformer ladder. When considering the phase and magnitude variations of each cell, the output power is

$$
P_{\text {OUT }}=\frac{1}{2 R_{L}}\left|\sum_{i=1}^{n} \frac{\eta_{i} \exp \left(j \theta_{i}\right) A_{v} V_{\text {in }}}{n}\right|^{2} .
$$

Dividing (11) with the output power of the ideal case, the overall combination efficiency due to phase and magnitude variation of various cells is expressed as

$$
\eta_{\text {combine }}=\left|\sum_{i=1}^{n} \frac{\eta_{i} \exp \left(j \theta_{i}\right)}{n}\right|^{2} .
$$

Since the focus of the analysis is on the phase and magnitude differences between the different cells, it can be assumed that losses that are common to each amplifier cell is incorporated 
into the amplifier gain $A_{v}$, therefore, the magnitude factors of each cell $\eta_{i}$ should be normalized to an average of unity

$$
\frac{1}{n} \sum_{i=1}^{n} \eta_{i}=1
$$

If the loss along the transformer ladder is to be discussed, then the constraint of (13) should not be used, and then the magnitude factors should be normalized such that the largest $\eta_{i}$ is unity.

Defining $P_{\mathrm{dc}}$ as the dc power of each cell and $\eta_{\mathrm{DE}}$ as the drain efficiency of the cell, an $n$-cell stack should deliver more RF power than an $(n-1)$-cell stack; hence,

$$
\begin{gathered}
P_{\mathrm{dc}} \cdot n \cdot \eta_{\mathrm{DE}} \cdot \eta(n)>P_{\mathrm{dc}} \cdot(n-1) \cdot \eta_{\mathrm{DE}} \cdot \eta(n-1) \\
\eta(n)>\eta(n-1) \cdot\left(1-\frac{1}{n}\right) .
\end{gathered}
$$

The factor $(1-1 / n)$ represents the incremental efficiency drop ratio from an $(n-1)$-cell stack to an $n$-cell stack. Since in reality the combination efficiency drops as the number of cell increases, and $(1-1 / n)$ approaches unity as $n$ increases, there is an upper limit to the number of cells that can be stacked.

First consider the case where there is only a magnitude variation among the transistor cells caused by the slightly different voltage gain of each amplifier cell, and negligible loss in the transformer ladder. Setting the cell number $n$ as a constant, the magnitude factors can be normalized to an average magnitude factor of 1 , it is clear that the overall combination efficiency is unaffected by such magnitude variation.

In the case where the magnitude steadily decreases along the transformer ladder due to ohmic loss and radiation, the outputs of the amplifier cells become steadily smaller. In this case, the cells farther down the transformer ladder contribute less to the overall output, and the efficiency drops as the number of cells increases. From (12) and assuming that the loss at each transformer is equal, the power combination efficiency of the stack of an $n$-cell stack is

$$
\eta_{\text {combine }}=\left|\sum_{i=1}^{n} \frac{\eta}{n}\right|^{2}=\left(\frac{\eta\left(1-\eta^{n}\right)}{n(1-\eta)}\right)^{2} .
$$

In (15), $(1-\eta)$ represents the voltage loss of each transformer. Applying (15) to the incremental efficiency drop constraint of (14) will give the upper limit of the number of cells at various voltage loss

$$
\left(\frac{n-1}{n}\right)\left(\frac{1-\eta^{n}}{1-\eta^{n-1}}\right)^{2}>1
$$

Using (15), the power combination efficiency versus the number of cells with different transformer efficiencies are plotted in Fig. 5, and (16) dictates the upper cell limit at various transformer efficiencies. For example, the upper cell limit for efficiencies of 0.9 and 0.8 are 12 and 6, respectively. From Fig. 5, the transformer loss should be extremely low (with efficiency of at least 0.99 , an approximately $0.09-\mathrm{dB}$ loss) for an

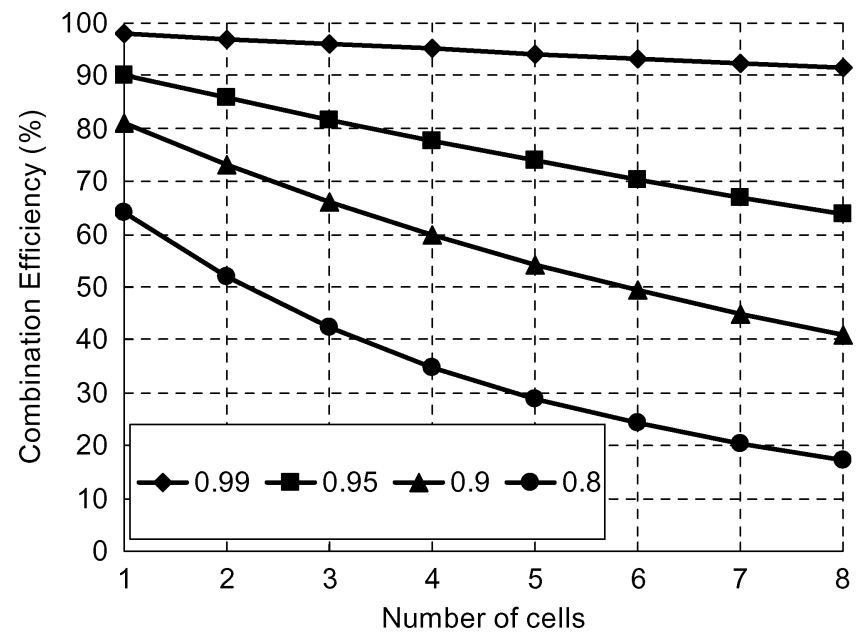

Fig. 5. Power combination efficiency of a stacked amplifier with lossy transformers in each cell.

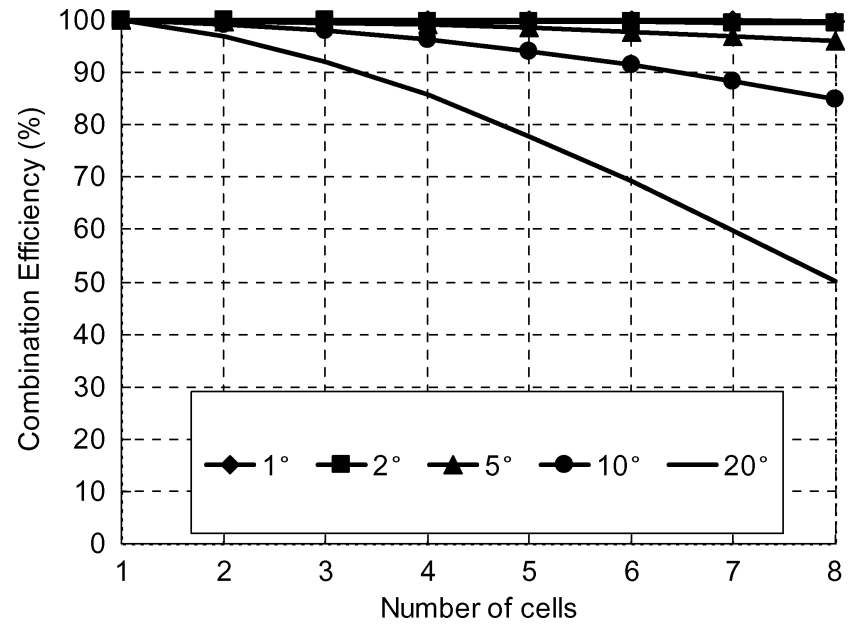

Fig. 6. Power combination efficiency of a stacked amplifier with increasing phase delay in each cell.

efficient combination efficiency of over $90 \%$ up to eight cells; for a four-cell stack, the transformer should have an efficiency of at least 0.95 (0.45-dB loss).

The distribution nature of the transformer components will result in an increasing phase delay along the transformer ladder. Assuming that the magnitude factor of each cell is unity, and a delay of $\theta$ between adjacent cells, from (11) the power combination efficiency is

$\eta_{\text {combine }}=\left|\sum_{k=1}^{n} \frac{\exp (j k \theta)}{n}\right|^{2}=\left\{\begin{array}{l}\frac{\sin ^{2}(n \theta / 2)}{n^{2} \sin ^{2}(\theta / 2)}, \quad \theta \neq 0 \\ 1, \quad \theta=0 .\end{array}\right.$

The incremental efficiency limit is

$$
\left(\frac{n-1}{n}\right)\left(\frac{\sin (n \theta / 2)}{\sin ((n-1) \theta / 2)}\right)^{2}>1
$$

The power combination efficiency versus number of cells with different phase delays are plotted in Fig. 6 using (17). The effect of phase delay on the cell-number limit is more tolerant 


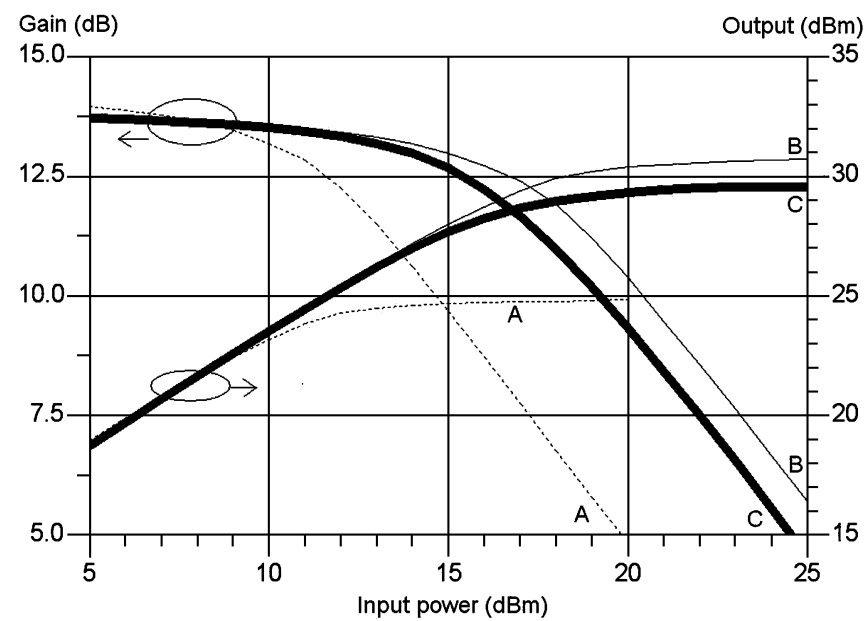

Fig. 7. $P_{\text {out }}$ versus $P_{\text {in }}$ and gain versus $P_{\text {in }}$ relationships of an ideal amplifier cell (A), four ideal amplifier cells stacked in series (B), and a four-cell stack with distributed effects considered (C).

than transformer loss: with $10^{\circ}$ of incremental delay, the cell limit is 13 , and for $20^{\circ}$ of delay, the cell limit is seven. A four-cell stack is quite tolerable to large phase delay, with still over $85 \%$ combining efficiency when adjacent cells delay each other by $20^{\circ}$. For eight cells, the requirement for efficient combining efficiency is more stringent, a $20^{\circ}$ phase delay will result in only $50 \%$ efficiency, which is only as good as four ideal devices, and in essence, poorer than a seven-cell stack from the constraint of (18). A graphic representation of this concept was presented in [15].

\section{Effects of Cell Imbalance on Amplifier Linearity}

For any solid-state amplifier, its output power versus input power can be characterized by a three-segment line, i.e., the linear region, compression region, and saturation region. This input power and output power relationship of an amplifier is usually measured or simulated using continuous wave $(\mathrm{CW})$ signals, and plotted as a $P_{\text {out }}-P_{\text {in }}$ chart, both in decibel scale. A two-tone $\mathrm{CW}$ measurement can provide further insights into amplifier linearity from the third-order intermodulation product it generates. A simulated $P_{\text {out }}-P_{\text {in }}$ relationship of a single device transformer-coupled amplifier with lossless matching is plotted in Fig. 7, dashed lines denoted by symbol "A."

The stacked amplifier is composed of several amplifier cells, each cell with a transistor that has a $P_{\text {out }}-P_{\text {in }}$ relationship mentioned above. In the ideal case where all the cells are identical and driven equally with the same magnitude and phase, the $P_{\text {out }}-P_{\text {in }}$ relationship of the stack amplifier will be exactly the linear combination of the individual amplifier cells. Using the ideal transformer-coupled amplifier characteristics of Fig. 7 "A" as the example, a four-cell ideal stack will result in the $P_{\text {out }}-P_{\text {in }}$ lines extended by $6 \mathrm{~dB}$, represented by thin solid lines denoted "B," and an eight-cell ideal stack will extend by $9 \mathrm{~dB}$. In other words, the output power will increase $n$ fold, with no degradation in linearity.

However, the distributed nature of the actual physical components will affect the linearity of the amplifier. Once the ideal transformers are replaced by coupled lines with distributed effects and connections between transistors replaced by transmis- sion lines, the power performance will degrade with poorer saturation power and earlier compression. The bold lines marked " $C$ " represent the four-cell stack with physical components. The output power is degraded by $1 \mathrm{~dB}$ compared to the ideal four-cell case, as well as earlier gain rolloff. Additional distributed effects in the cells, such as the connections to the transformers, metal-insulator-metal (MIM) capacitors, and the structure of the device will further affect the linearity performance, which are observed in the experiments.

The reason to the change in the linearity of the stacked amplifier may be explained as follows: the input feeds into the cells are not ideal; therefore, the input signals into each cell may be slightly varied both in magnitude in phase. When the input power is low and all the devices are operating in the linear region, the devices all act as linear amplifiers. As the input power increases, the devices that have a slightly higher proportion of input power will enter compression faster, and the devices with lower proportion of input power will still be in the linear operating region. Such a division of operating modes will cause the entire stack to operate at an under-par condition. The overall effect is that the stacked amplifier enters compression earlier than the ideal case.

The circuit size should therefore be as compact as possible to mitigate the distributed effects on power performance of the amplifier.

\section{FOUR-CELL HBT PA DESIGN}

With the stacked amplifier configuration, one has the option of power combination by increasing voltage, adding an additional degree of design freedom. Depending on the system requirements and design constraint, to obtain more power, one may stack many amplifier cells at the expense of higher bias voltage, or to increase device periphery and stacking at the same time to maintain constant optimum load impedance. With the second approach, one should choose to keep the optimum load admittance slightly lower than $1 / 50 \mathrm{~S}$, thus making the amplifier easy to match with a high-pass short stub.

The series-input stack also has the additional advantage of increasing input impedance, as proven in Section II, which means that the coupling transformers do not need to be designed as impedance transformation components, as the overall input impedance with simple $1: 1$ transformers will still be relatively easy to match. With the optimum load impedance designed around $50 \Omega$, with the series-input approach, the input impedance will also be around the center of the Smith chart.

With the design constraint of output power $P_{\text {out }}$ and optimum load impedance of $50 \Omega$, the design flow of a series-input and series-output transformer coupled stacked amplifier is summarized as follows.

1) From the power equation $P_{\text {out }}=n^{2}\left(V_{d d}-V_{k}\right)^{2} /\left(2 \times R_{L}\right)$, where $V_{d d}$ is the drain/collector bias of a single device, $V_{k}$ is the knee voltage of a single device, and $R_{L}$ is the desired optimum load impedance of $50 \Omega$; the number of cells $n$ can be solved to the closest integer.

2) The optimum load impedance of each cell is $R_{L} / n$, based on this, the device size is decided.

3) Assemble each cell: device, transformer, biasing, and bypass capacitors. Component sizes and losses should be kept 


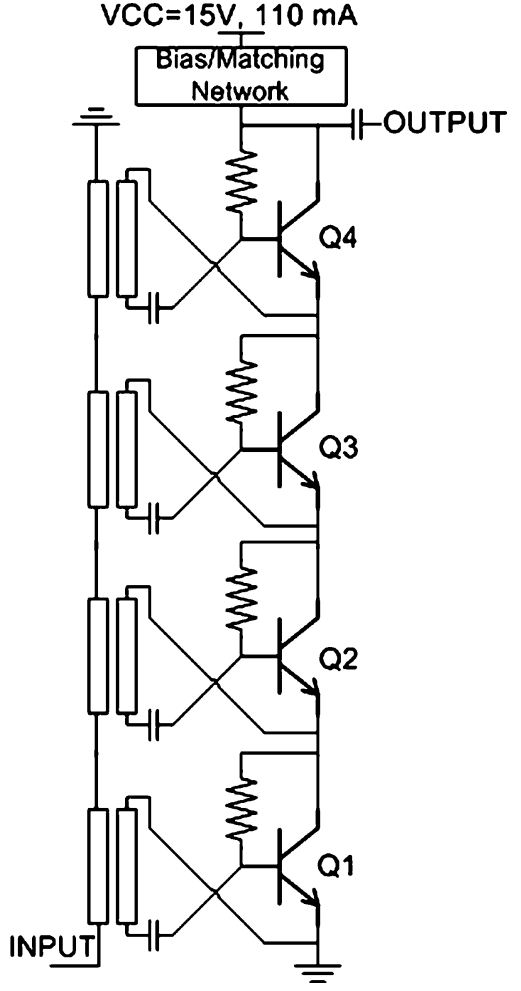

Fig. 8. Simplified schematic of the HBT series-input stack amplifier.

to a minimum to reduce the nonideal effects. Care should be taken at the layout so that each cell can be easily connected with each other both at the input and output end.

4) Combine the cells; design the output matching network to its optimum load impedance, and biasing circuits.

5) Design the input matching circuit.

To validate the series-input and series-output stacked amplifier, a four-cell stack amplifier operating at $5-6 \mathrm{GHz}$ with $1-\mathrm{W}$ saturation power is designed using WIN Semiconductors' $2-\mu \mathrm{m}$ HBT process. ${ }^{1}$ A major feature of the series-input stack is that the input impedance also increases with the number of cells; therefore, it is possible to make both the input impedance and output optimum load to near $50 \Omega$ at the same time to simplify the matching designs.

A simplified schematic, excluding the matching and biasing circuits of the series-input stack amplifier, is shown in Fig. 8. It is composed of four identical self-biased HBT devices in series and coupled lines as the transformer coupler. Series capacitors are placed between the base and emitter of each transistor to serve as dc blocks. Due to the layout configuration of the HBT device, the crossover between the transformer and base-emitter represents how the coupled-line transformer actually connects to the device, instead of an actual crossover. Such a connection will change the direction of the current in the output coil of the linear analysis in Section II, which will alter the (2)-(12) slightly, but with the same advantages of increased input impedance, output impedance, and power combination. The input is fed from the bottom up, instead of top down in the analysis, allowing easier layout of the RF and dc pads.

\footnotetext{
${ }^{1}$ Online information of process at http://www.winfoundry.com
}

TABLE II

Magnitude AND Phase ERROR of the INPUt Coupled LINE LADDER, RELATIVE TO Q1

\begin{tabular}{lcc}
\hline \hline Device & Phase difference (degrees) & Magnitude Difference (dB) \\
\hline Q2 & 1.72 & 0.284 \\
Q3 & 2.14 & 0.353 \\
Q4 & 2.44 & 0.36 \\
\hline \hline
\end{tabular}

The device used is seven single two-finger $20-\mu \mathrm{m}$ devices combined in parallel with a 3- $\Omega$ emitter ballast resistor in each cell to reduce the multifinger emitter collapse phenomenon [18]. A voltage level of $3.75 \mathrm{~V}$ and quiescent current of $110 \mathrm{~mA}$ is chosen for the design. This sets the voltage of the entire amplifier at $15 \mathrm{~V}$.

Due to the particular layout of the transistor, the bottom transistor Q1 has a slightly different layout compared to the other three cells; this, in turn, causes a slight difference in the input feed signal of Q1. In fact, the magnitude of the Q1 feed is smaller than the other three cells. The phase and magnitude difference of the input coupled line ladder, relative to the first device Q1, is listed in Table II. Based on (17) and Fig. 6, the phase difference has a minimal effect on the combination efficiency of at most $2 \%$. However, the asymmetric layout does affect the linearity performance, which is demonstrated in Section II-C. This is also observed from the load lines. Fig. 9(a) shows the load lines of the individual transistors in an ideal simulation (lumped components only) at $25-\mathrm{dBm}$ output power; it is clear that the four load lines have the same shape and are nearly identical in size. However, once all of the components are replaced with EM simulations, the load lines of the transistors degrade to the contours shown in Fig. 9(b); in particular, the load line of Q1 is distinctively different from the other three, which is the manifestation of the different layout in Q1. The voltage clipping of Q1 in Fig. 9(b) is also evidence that Q1 is the dominant factor in the earlier compression of the stacked amplifier in power measurements.

The transformer couplers are implemented using coupled lines. The lines are $290 \mu \mathrm{m}$ in length, linewidth of $5 \mu \mathrm{m}$, and gap of $2 \mu \mathrm{m}$. The length of the coupled line is very short compared to a quarter-wavelength line at $5 \mathrm{GHz}$, and because of its thin linewidth and the small spacing, the coupling is mostly magnetic, which can be modeled as a transformer in Fig. 2(a). Based on EM simulations, the resistance in each inductor is $2 \Omega$, the self-inductance $L_{1}$ is $0.32 \mathrm{nH}, L_{2}$ is $0.23 \mathrm{nH}$, and the mutual inductance $M_{1}$ is $0.19 \mathrm{nH}$.

Circuit simulations are conducted using Agilent's ADS software, and electromagnetic (EM) simulations using SONNET [17]. Load-pull simulations at $5 \mathrm{GHz}$ are conducted to determine the optimum load impedance. The output power, optimum load impedance, and gamma of the device are listed in Table III with respect to the number of stacked cells. The result is consistent with the stack amplifier theory with the optimum load impedance and output-power in proportion to the number of devices stacked. The input impedance of the amplifier also increased accordingly to the number of cells stacked. The four-cell input impedance is around $12.5 \Omega$, which is over a fourfold increase of $2.5 \Omega$ of a single transistor. 


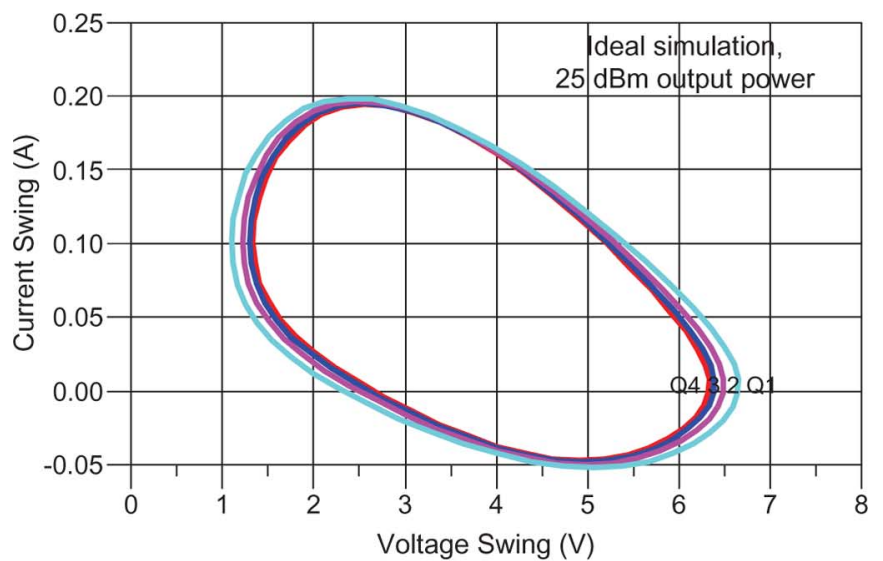

(a)

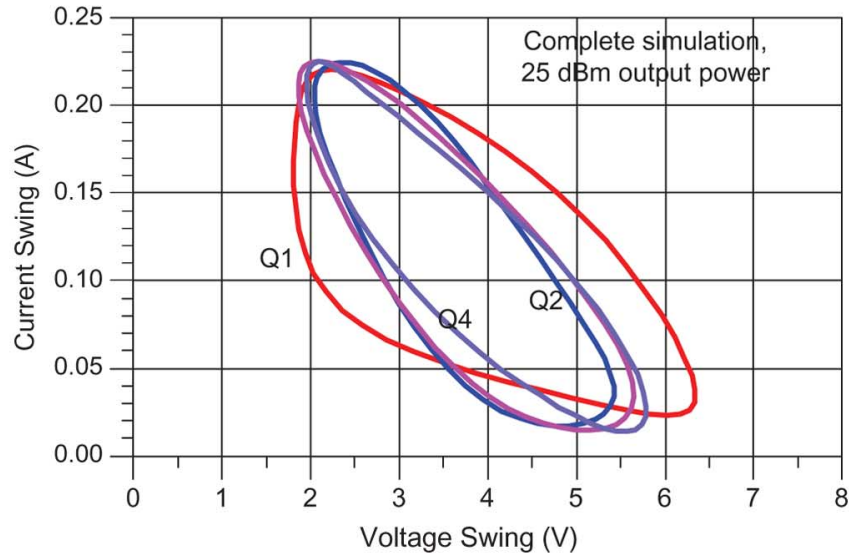

(b)

Fig. 9. Load line of the four transistors. (a) Load lines under ideal simulations. (b) Load lines under complete simulation.

\section{TABLE III}

OUtPut POWER, OPTIMUM LOAD IMPEDANCE, AND GAMMA VERSUS NUMBER OF CELLS

\begin{tabular}{lcccc}
\hline \hline Cells & $\mathrm{P}_{\text {in }}(\mathrm{dBm})$ & $\mathrm{P}_{\text {out }}(\mathrm{dBm})$ & $\begin{array}{c}\text { Impedance } \\
\text { (normalized) }\end{array}$ & Gamma \\
\hline 1 & 18 & 24.17 & $0.307+\mathrm{j} 0.185$ & $0.543 \angle 156.9^{\circ}$ \\
2 & 21 & 27.15 & $0.617+\mathrm{j} 0.282$ & $0.290 \angle 133.7^{\circ}$ \\
4 & 24 & 29.9 & $1.118+\mathrm{j} 0.497$ & $0.235 \angle 63.4^{\circ}$ \\
\hline \hline
\end{tabular}

A chip photograph of the stacked PA is shown in Fig. 10 with a chip size of $2 \times 1 \mathrm{~mm}^{2}$. From Fig. 10, the input matching occupies very little chip area, and the output matching is much simpler than most PA output matches. Details of a single cell are shown in Fig. 11, which shows the orientation of the transistor, coupled line, dc block, input matching circuits, and several crossovers of the Q1 transistor. Due to the peculiar layout, the right end of the coupled line, which should be a dc and RF short, is far away from the via-hole ground, therefore, an additional via-hole is added, denoted as "Enforce emitter ground." This additional via-hole is only added to the Q1 cell, therefore making Q1 different in layout from the other three cells, resulting in different large-signal performance.

\section{Measurement Results}

The series-input series-output amplifier is measured on-wafer. The $S$-parameter is measured using the Anritsu

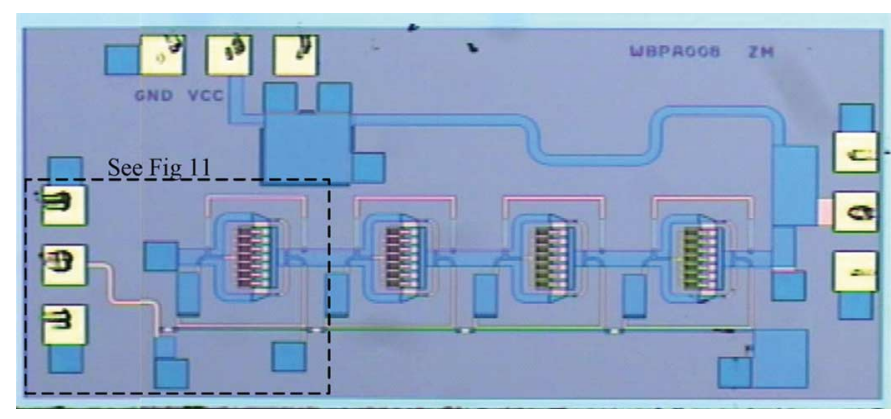

Fig. 10. Chip photograph of the series-input series-output transformer coupled stacked PA. The chip size is $2 \times 1 \mathrm{~mm}^{2}$.

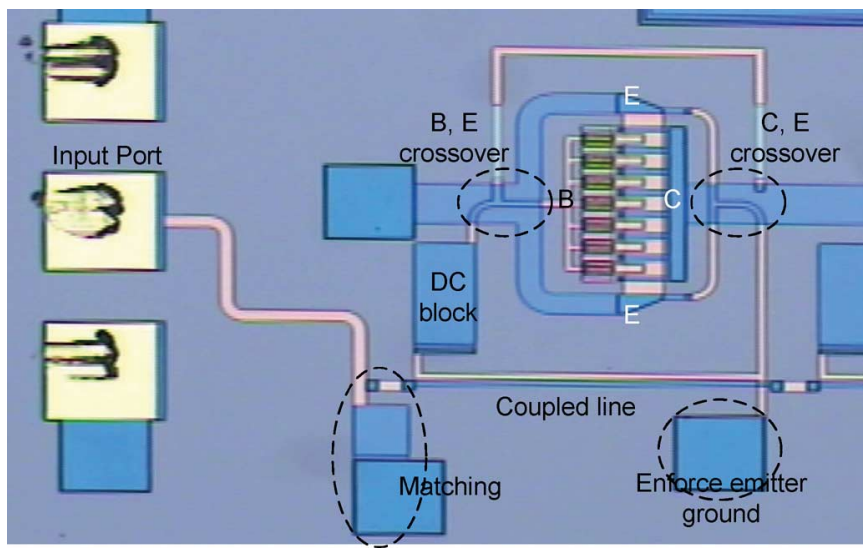

Fig. 11. Detailed photograph of the input port and the first cell. The emitter, base, and collectors of the transistor are denoted as "E," "B," and "C," respectively.

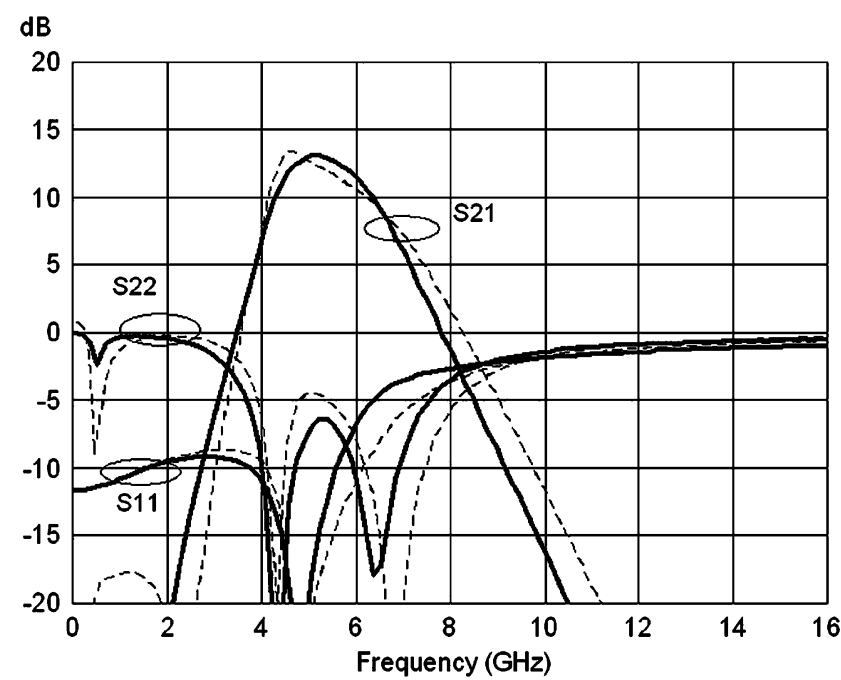

Fig. 12. $S$-parameter measurements of the four-cell stack PA. Bias voltage is $15 \mathrm{~V}$ and quiescent current is $110 \mathrm{~mA}$. The solid lines are measurements and the dashed lines are the simulation results.

37397D vector network analyzer. The $S$-parameter measurements up to $16 \mathrm{GHz}$ are shown in Fig. 12. The amplifier has 13.1-dB gain, 6-dB input return loss, and 16-dB output return loss at $5 \mathrm{GHz}$. The best input return loss is at $4.8 \mathrm{GHz}$ of $28.7 \mathrm{~dB}$, and over 12-dB gain from 4.6 to $5.8 \mathrm{GHz}$. The dotted lines are the simulation results, and accurately predict the gains and return losses of the amplifier. 


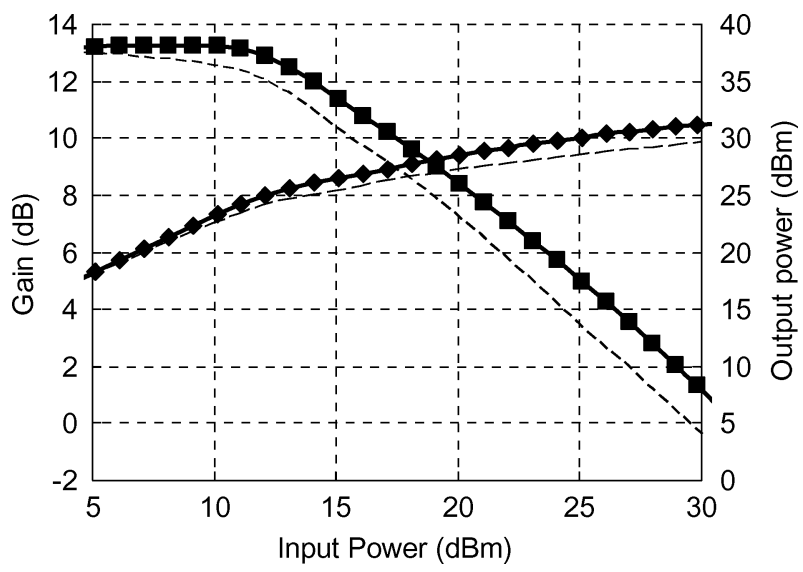

Fig. 13. Power measurements of the four-cell stack amplifier at $4.8 \mathrm{GHz}$. The solid lines are measurements and the dashed lines are the simulation results.

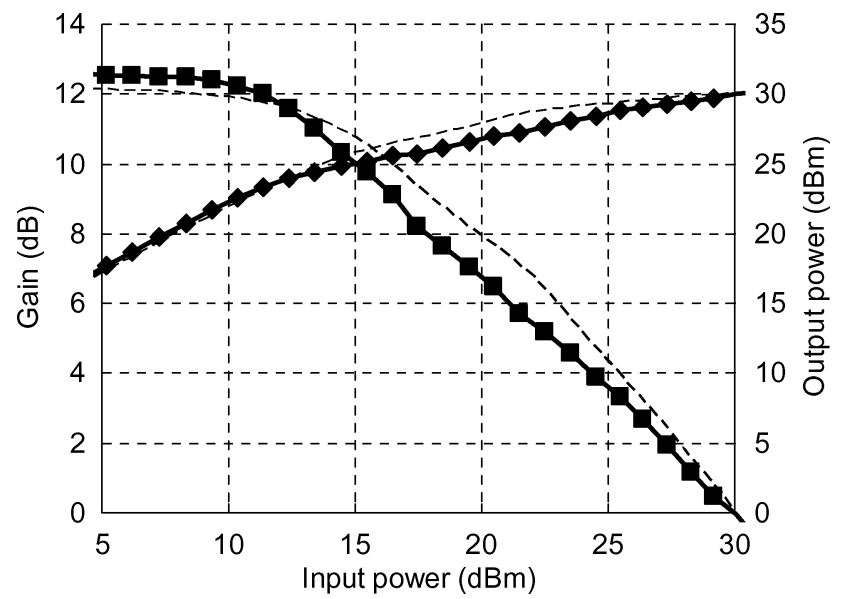

Fig. 14. Power measurements of the four-cell stack amplifier at $5.2 \mathrm{GHz}$. The solid lines are measurements and the dashed lines are the simulation results.

Power measurements are measured using a signal source and an HP 83020 PA as input, and an HP 8481B power sensor for output power measurements. Power measurements at 4.8 and $5.2 \mathrm{GHz}$ are measured. Figs. $13-15$ show the $P_{\text {out }}-P_{\text {in }}$ characteristics of the amplifier at 4.8,5.2, and 5.8 GHz, respectively. The large-signal gain of the amplifier at each frequency is also plotted in the corresponding graphs. The output $1-\mathrm{dB}$ compression power at $4.8 \mathrm{GHz}$ is $26 \mathrm{dBm}$, at $5.2 \mathrm{GHz}$ is $23.4 \mathrm{dBm}$, and at $5.8 \mathrm{GHz}$ is $20 \mathrm{dBm}$. The measured power-added efficiency is shown in Fig. 16. The simulation has predicted the early gain compression due to the distributed effects of the components, which may be observed in Figs. 13-15 as a bend in output power around $10-15-\mathrm{dBm}$ input power.

The four-cell stack amplifier is subjected to actual 802.11a modulation signals at 5.2 and $5.8 \mathrm{GHz}$. The input signals are generated using an Agilent E4438C vector signal generator, and the output signals are received and demodulated using an Agilent $89605 \mathrm{~B}$ vector signal analyzer. The output spectrum of the four-cell stack amplifier is shown in Fig. 17. The output power is $+18 \mathrm{dBm}$ with a $54-\mathrm{Mb} / \mathrm{s}$ rate signal at $5.2-\mathrm{GHz}$ carrier frequency. The spectrum is within the spectral mask defined in the 802.11a standard. The error vector magnitude (EVM) versus

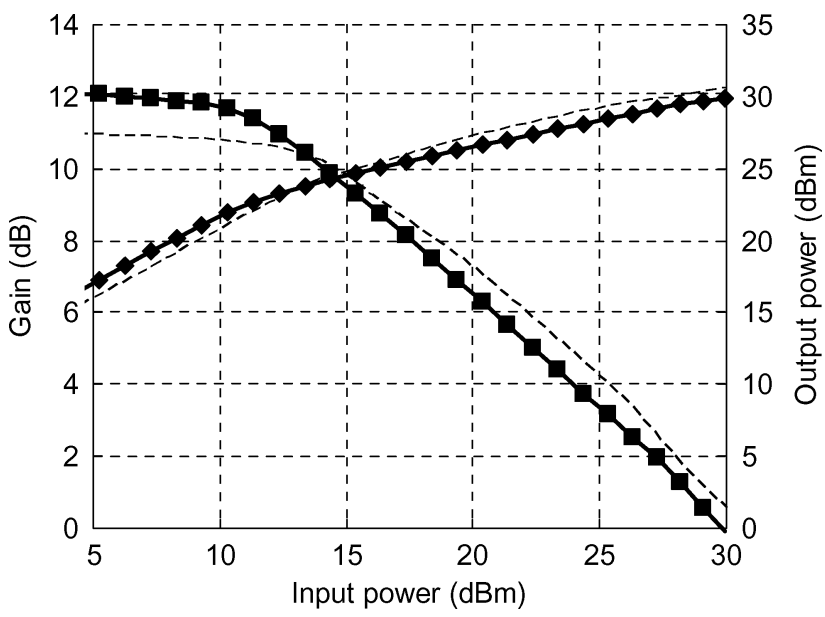

Fig. 15. Power measurements of the four-cell stack amplifier at $5.8 \mathrm{GHz}$. The solid lines are measurements and the dashed lines are the simulation results.

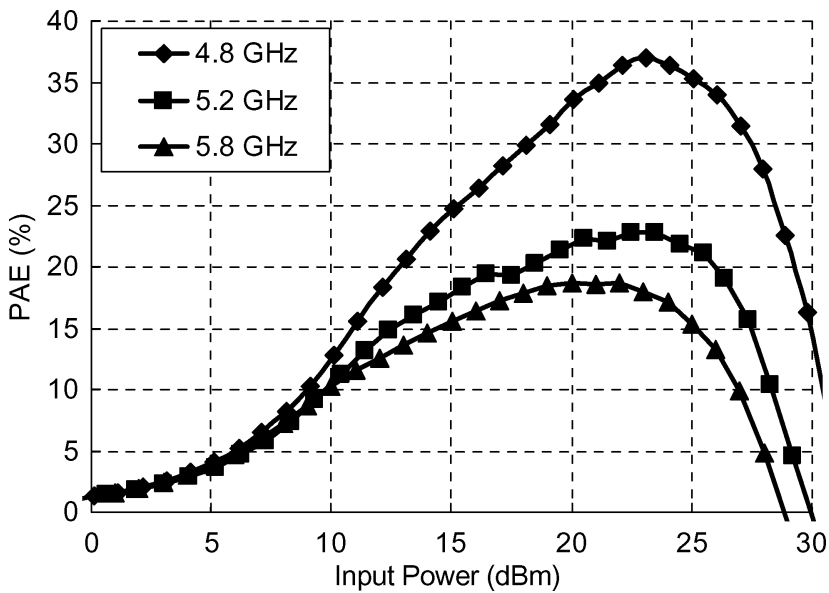

Fig. 16. Measured power-added efficiency at $4.8,5.2$, and $5.8 \mathrm{GHz}$

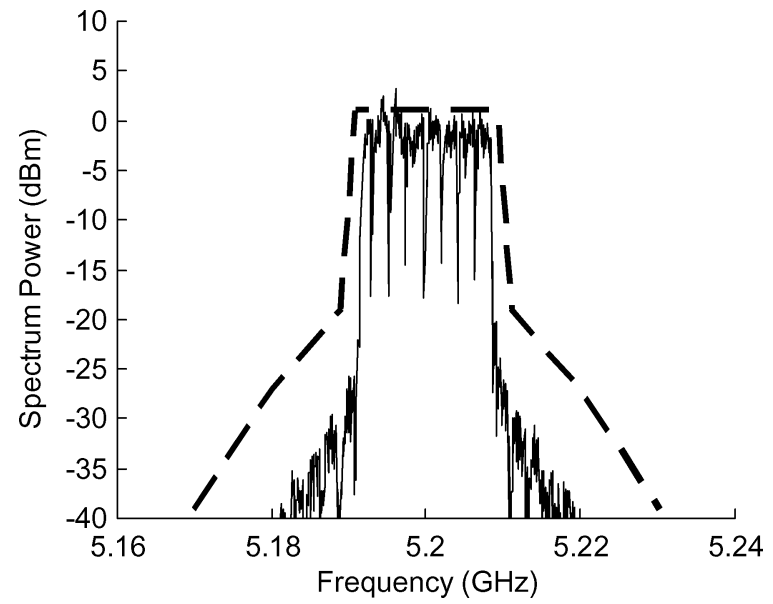

Fig. 17. Measured PA spectrum at $5.2 \mathrm{GHz}$ subjected to $54-\mathrm{Mb} / \mathrm{s}$ signal with an output power of $18 \mathrm{dBm}$.

output power is shown in Fig. 18 with a 54-Mb/s data rate signal. The EVM requirement of $-25 \mathrm{~dB}$ can be achieved while delivering $+18 \mathrm{dBm}$ of output power at 5.2 and $5.8 \mathrm{GHz}$. The adjacent channel power ratio (ACPR) at the two frequencies 


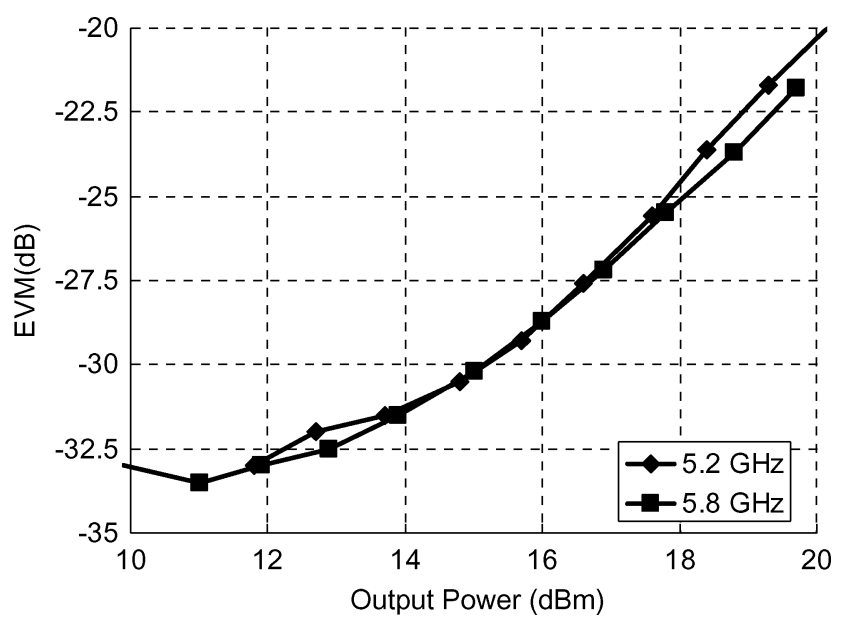

Fig. 18. EVM versus transmission power at $54-\mathrm{MB} / \mathrm{s}$ rate at carrier frequencies of 5.2 and $5.8 \mathrm{GHz}$.

at $+18-\mathrm{dBm}$ output power at 5.2 and $5.8 \mathrm{GHz}$ are better than $30 \mathrm{~dB}$.

The power performance is degraded from the ideal case due to the various distributed effects of the connecting lines and passive components. To improve these effects, there are several areas for improvement. One place is to reduce the size of the coupled line ladder. From the chip photograph in Fig. 10, there seems to be ample area for the reduction of the coupled line ladder without interfering with the devices. The coupled lines can be reduced in size by meandering the lines. Resimulation on the input feed lines shows that a $20 \%$ reduction in length will have only $0.15-\mathrm{dB}$ error, and $40 \%$ reduction will reduce the magnitude imbalance to negligible levels. The reduction in the line lengths between the transistors will also improve the circuit power performance. Due to the particular device layout, the bottom device connection to ground results in the bottom cell to be slightly different from the other three cells. This is also a source of imbalance that causes the linearity of the amplifier to degrade. Improvements on the bottom cell layout will also improve the circuit's power performance.

\section{CONCLUSION}

The design and analysis of the series-input and series-output stacked PA has been reported in this paper. This configuration is rigorously analyzed, proving that the series input has the additional advantage of increased input impedance. The relationship between the feed balance and the overall combination efficiency can be described analytically with several cases specifically discussed. The physical connections will affect the power performance of the stacked amplifier, and is also discussed. A four-cell stacked amplifier is demonstrated using a $2-\mu \mathrm{m}$ HBT process. Measurements demonstrate over 12-dB gain from 4.6 to $5.8 \mathrm{GHz}, 1-\mathrm{dB}$ compression power of $26 \mathrm{dBm}$ at $4.8 \mathrm{GHz}$, and $23.4 \mathrm{dBm}$ at $5.2 \mathrm{GHz}$. The four-cell stacked amplifier is subjected to 802.11a modulation signals, conforming to EVM requirements at $+18-\mathrm{dBm}$ output and $54-\mathrm{Mb} / \mathrm{s}$ data rate, and ACPR of better than $30 \mathrm{~dB}$.

\section{ACKNOWLEDGMENT}

The authors wish to thank Dr. C.-H. Wang and Dr. J.-H. Tsai, both with National Taiwan University, Taipei, Taiwan, R.O.C., for their helpful comments, Prof. H.-Y. Chang, National Central University, Jhongli City, Taoyuan County, Taiwan, R.O.C., for his assistance in measurements, and Dr. C.-C. Chiong, Academia Sinica Institute of Astronomy and Astrophysics (ASIAA), Taipei, Taiwan, R.O.C., for his coordination support.

The chip was fabricated by WIN Semiconductors, Tao Yuan County, Taiwan, R.O.C., with the support of the ASIAA.

\section{REFERENCES}

[1] G. Gonzalez, Microwave Transistor Amplifiers, Analysis and Design, 2nd ed. Upper Saddle River, NJ: Prentice-Hall, 1997, ch. 3.

[2] S. C. Cripps, RF Power Amplifiers for Wireless Communications. Boston, MA: Artech House, 1999.

[3] T. Sowlati and D. M. W. Leenaerts, "A 2.4-GHz 0.18- $\mu \mathrm{m}$ CMOS selfbiased cascode power amplifier," IEEE J. Solid-State Circuits, vol. 38, no. 8, pp. 1318-1324, Aug. 2003.

[4] C. Park, Y. Kim, H. Kim, and S. Hong, "A 1.9-GHz CMOS power amplifier using three-port asymmetric transmission line transformer for a polar transmitter," IEEE Trans. Microw. Theory Tech., vol. 55, no. 2, pp. 230-238, Feb. 2007.

[5] A. Mazzanti, L. Larcher, R. Brama, and F. Svelto, "Analysis of reliability and power efficiency in cascode class-E PAs," IEEE J. Solid-State Circuits, vol. 41, no. 5, pp. 1222-1229, May 2006

[6] C.-M. Lo, C.-S. Lin, and H. Wang, "A miniature $V$-band 3-stage cascode LNA in $0.13 \mu \mathrm{m}$ CMOS," in IEEE Int. Solid-State Circuits Conf. Dig., Feb. 2006, pp. 322-323, 656

[7] M. Shifrin, Y. Ayasli, and P. Katzin, "A new power amplifier topology with series biasing and power combining of transistors," in IEEE Microw. Millimeter-Wave Monolithic Circuits Symp. Dig., Jun. 1-3, 1992, pp. 39-41.

[8] A. Ezzeddine, H.-L. A. Hung, and H. C. Huang, "High-voltage FET power amplifiers for satellite and phased-array applications," in IEEE MTT-S Int. Microw. Symp. Dig., Jun. 1985, no. 1, pp. 336-339.

[9] A. K. Ezzeddine and H. C. Huang, "The high voltage/high power FET (HiVP)," in IEEE RFIC Symp. Dig., Jun. 2003, pp. 215-218.

[10] A. K. Ezzeddine and H. C. Huang, "Ultra-broadband GaAs HIFET MMIC PA," in IEEE MTT-S Int. Microw. Symp. Dig., Jun. 2006, pp. $1320-1323$.

[11] J. Jeong, S. Pornpromlikit, P. M. Asbeck, and D. Kelly, “A $20 \mathrm{dBm}$ linear RF power amplifier using stacked silicon-on-sapphire MOSFETs," IEEE Microw. Wireless Compon. Lett., vol. 16, no. 12, pp. 684-686, Dec. 2006.

[12] A. Ezzedine and H. C. Huang, "High power high impedance microwave devices for power applications," U.S. Patent 6 137367, Oct. 24, 2000.

[13] J. G. McRory, G. G. Rabjohn, and R. H. Johnston, "Transformer coupled stacked FET power amplifiers," IEEE J. Solid-State Circuits, vol. 34, no. 2, pp. 157-161, Feb. 1999.

[14] G. G. Rabjohn, M. S. Suthers, J. McRory, and R. Leroux, "Transformer coupled stacked power amplifier,” U.S. Patent 5867 061, Feb. 2, 1999.

[15] Z.-M. Tsai, M.-F. Lei, and H. Wang, "An HBT four-cell monolithic stacked power amplifier," in IEEE MTT-S Int. Microw. Symp. Dig., Jun. 3-8, 2007, pp. 151-154.

[16] “Advanced Design System documentation 2005A," Agilent Technol., Palo Alto, CA, 2005.

[17] “Sonnet Users Guide, Release 9," Sonnet Software Inc., Pittsburgh, PA, 2003.

[18] J. S. Yuan, SiGe, GaAs, and InP Heterojunction Bipolar Transistors. New York: Wiley, 1999, pp. 306-319.

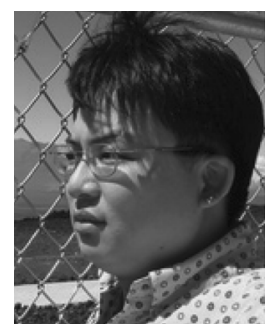

Ming-Fong Lei (S'00) was born in Taipei, Taiwan, R.O.C., on December 20, 1980. He received the B.S. degree in electrical engineering and $\mathrm{Ph} . \mathrm{D}$. degree in communication engineering from National Taiwan University, Taipei, Taiwan, R.O.C., in 2002 and 2007, respectively.

His research interests include the design and analysis of microwave and millimeter-wave circuits, microwave device modeling, and filters. 


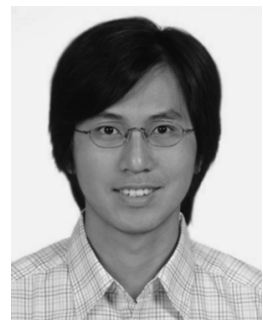

Zuo-Min Tsai (S'03-M'06) was born in Mailo, Taiwan, R.O.C., in 1979. He received the B.S. degree in electronic engineering and Ph.D. degree in communication engineering from National Taiwan University, Taipei, Taiwan, R.O.C., in 2001 and 2006, respectively.

He is currently a Post-Doctoral Research Fellow with the Graduate Institute of Communication Engineering, National Taiwan University. His research interests are the theory of microwave or millimeterwave circuits.

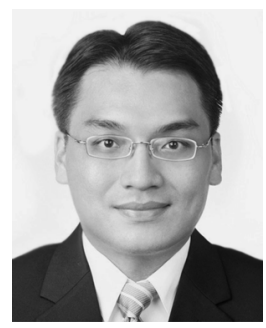

Kun-You Lin (S'00-M'04) was born in Taipei, Taiwan, R.O.C., in 1975. He received the B.S. degree in communication engineering from National Chiao Tung University, Hsinchu, Taiwan, R.O.C., in 1998, and the Ph.D. degree in communication engineering from National Taiwan University, Taipei, Taiwan, R.O.C., in 2003.

From August 2003 to March 2005, he was a Post-Doctoral Research Fellow with the Graduate Institute of Communication Engineering, National Taiwan University. From May 2005 to July 2006, he was an Advanced Engineer with the Sunplus Technology Company Ltd., Hsin-Chu, Taiwan, R.O.C. In July 2006, he joined the faculty of the Department of Electrical Engineering and Graduate Institute of Communication Engineering, National Taiwan University, as an Assistant Professor. His research interests include the design and analysis of microwave/RF circuits.

Dr. Lin is a member of Phi Tau Phi .

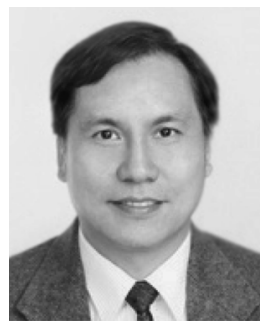

Huei Wang (S'83-M'87-SM'95-F'06) was born in Tainan, Taiwan, R.O.C., on March 9, 1958. He received the B.S. degree in electrical engineering from National Taiwan University, Taipei, Taiwan, R.O.C., in 1980, and the M.S. and Ph.D. degrees in electrical engineering from Michigan State University, East Lansing, in 1984 and 1987, respectively.

During his graduate study, he was engaged in research on theoretical and numerical analysis of EM radiation and scattering problems. He was also involved in the development of microwave remote detecting/sensing systems. In 1987, he joined the Electronic Systems and Technology Division, TRW Inc. He has been an MTS and Staff Engineer responsible for MMIC modeling of computer-aided design (CAD) tools, MMIC testing evaluation, and design and became the Senior Section Manager of the Millimeter-Wave (MMW) Sensor Product Section, RF Product Center. In 1993, he visited the Institute of Electronics, National Chiao-Tung University, Hsinchu, Taiwan, R.O.C., to teach MMIC-related topics. In 1994, he returned to TRW Inc. In February 1998, he joined the faculty of the Department of Electrical Engineering, National Taiwan University, as a Professor. He is currently the Director of the Graduate Institute of Communication Engineering, National Taiwan University.

Dr. Wang is a member of Phi Kappa Phi and Tau Beta Pi. He was the recipient of the Distinguished Research Award of National Science Council, R.O.C. (2003-2006). In 2005, he was elected as the first Richard M. Hong Endowed Chair Professor of National Taiwan University. He has been appointed an IEEE Distinguished Microwave Lecturer for the 2007-2009 term. 